\title{
Lost in re-election: a tale of two Spanish online campaigns
}

\author{
Helena Gallego ${ }^{1,2}$, David Laniado ${ }^{2}$, Andreas Kaltenbrunner ${ }^{1,2}$, Vicenç Gómez ${ }^{1}$, \\ and Pablo Aragón ${ }^{1,2}$ \\ 1 Universitat Pompeu Fabra, Barcelona, Spain, \\ 2 Eurecat - Technology Center of Catalonia, Barcelona, Spain
}

\begin{abstract}
In the 2010 decade, Spanish politics have transitioned from bipartidism to multipartidism. This change led to an unstable situation which eventually led to the rare scenario of two general elections within six months. The two elections had a mayor difference: two important left-wing parties formed a coalition in the second election while they had run separately in the first one. In the second election and after merging, the coalition lost around $1 \mathrm{M}$ votes, contradicting opinion polls. In this study, we perform community analysis of the retweet networks of the online campaigns to assess whether activity in Twitter reflects the outcome of both elections. The results show that the left-wing parties lost more online supporters than the other parties. Furthermore, we find that Twitter activity of the supporters unveils a decrease in engagement especially marked for the smaller party in the coalition, in line with post-electoral traditional polls.
\end{abstract}

Keywords: Twitter, Politics, Political Parties, Spanish Elections, Online Campaigning, Political Coalition, Engagement, Political Participation

\section{Introduction}

Social media are playing a key role in shaping public debate in political contexts, forming a new public sphere [6]; it is therefore increasingly important to understand their usage during political campaigns. Social media's function as a potential mirror of societal trends [5] and their strong impact on voters' perceptions and decision making make it important to understand their dynamics and influence [17, and their usage by politicians 1122. Although translating signals from the online to the offline world is not always straightforward, and previous studies aimed at predicting election results through the analysis of Twitter, e.g. 21, received many criticisms $7 / 11 / 15$, it is undoubted that the analysis of social media as emerging political battleground can unveil important aspects of electoral campaigns. Indeed, a growing amount of research is devoted in particular to investigate multiple aspects of the usage of Twitter during elections, as illustrated in the systematic literature review presented in [14.

In this study, we focus on the Spanish general elections of 2015 and 2016 and compare Twitter activity during the two consecutive campaigns to assess 
whether and how it reflects changes in the engagement of the supporters of different parties. This case study is of special interest because the 2015 general elections marked the end of forty years of Spanish bipartidism. After the country was shaken by the economic crisis of 2008 and by the $15 \mathrm{M}$ (or Indignados) movement of 2011, with massive protests against major traditional parties [19], the elections in December 2015 were held in a very different scenario with respect to all previous elections [18. The emergence of new political forces and the resulting fragmented parliament with no clear majority led, after six months of negotiation, to new elections in June 2016 [20].

The main parties involved in the elections and having a presence on the whole country are:

- Partido Popular ( $\mathrm{PP}^{3}$ - traditional, right);

- Partido Socialista Obrero Español (PSOE${ }^{4}$ - traditional, center/left);

- Izquierda Unida (IU ${ }^{5}$ - traditional, left);

- Podemos (Pod ${ }^{6}$ - new, left) ;

- Ciudadanos (CS7 new, center/right).

It is also important to mention the organizations Compromís (Valencia), En Marea (Galicia) and En Comú Podem (Catalunya), regional confluences which included local bottom-up forces in coalition with Podemos.

From the election results in Table B.3, we observe that the participation declined significantly from the first to the second election, suggesting a decrease in motivation of the electorate. They also show an increase in PP votes in 2016; this, combined with the participation drop, led to a significantly higher amount of representatives for this party. In the 2015 election, some of the main left parties ran together in a coalition formed by Podemos, En Comú Podem, Compromís and En Marea. Izquierda Unida decided to run alone, however in 2016 it joined the coalition which was re-named Unidos Podemos. The current Spanish electoral law, which penalizes small forces and gave IU only two representatives in the 2015 Congress after achieving almost one million votes, triggered the decision of the party to join the coalition in 2016. Although the sum of representatives of the coalition in 2016 was the same as the one achieved separately in 2015, the corresponding amount of votes dropped significantly (by around 1 million votes), contradicting several pre-election polls 8 .

While the electoral results clearly indicate an increase or decrease in votes of each party between the two elections, they do not explicitly indicate voter migration between parties (or between parties and abstentionism), which is left to opinion polls. Several studies using post and pre-electoral polls tried to determine the voter transfers from one election to the other. According to a study [16, $73 \%$ from UP repeated their vote and the rest abstained. Also [10] estimated that the coalition managed to retain $74 \%$ of Podemos voters, but only six out of

3 https://en.wikipedia.org/wiki/People\%27s_Party_(Spain)

4 https://en.wikipedia.org/wiki/Spanish_Socialist_Workers\%27_Party

5 https://en.wikipedia.org/wiki/United_Left_(Spain)

6 https://en.wikipedia.org/wiki/Podemos_(Spanish_political_party)

7 https://en.wikipedia.org/wiki/Citizens_(Spanish_political_party)

8 http://datos.cis.es/pdf/Es3141mar_A.pdf 
ten IU voters. This means that there were also differences in the distribution of voters within the coalition electorate. To complement opinion polls about voter migration between parties with evidence from social media activity, we formulate the following research question:

RQ1: Can we observe from Twitter activity a migration of supporters between parties from the first to the second election?

To answer this question, we consider the Twitter retweet network, perform a community analysis to identify clusters of political parties and characterize their structure following the methodology of [2]. As retweets generally represent endorsement, they have been proven useful in previous literature to detect clusters corresponding to political parties, both in the context of Spain [12] and other countries 8]. We will use the obtained clusters and study the migration of users between clusters. As we know from the electoral results that the parties who constituted Unidos Podemos lost more than 1 million voters, our hypothesis is that we will observe a drop in the users clustered around the accounts of these parties. We further expect the analysis to indicate which of the parties in the coalition lost most supporters in Twitter, and whether lost users started supporting other parties.

From 2015 to 2016, participation dropped significantly, showing a general demotivation or tiredness in the electorate. Several studies have examined the correlation between social media use and political engagement. Holt et al [12] report that both political social media usage and attention to political news in traditional media increase political engagement over time, and suggest that frequent social media use among citizens can function as a leveler in terms of motivating political participation. Findings from [3] reveal that a variety of Internet uses are positively related to different forms of political participation, whereas the relationship between most uses of traditional media and participation are weak. Finally, Dimitrova et al 9] report only weak effects of digital media use on political learning, but find that the use of some digital media forms has appreciable effects on political participation. As we know that Twitter activity can be related to political engagement and there has been a motivation decrease between the two campaigns, the second question of this study is:

RQ2: Is the demotivation of the electorate reflected in their Twitter activity/engagement?

We will answer this research question analyzing the volume of activity per user in the two campaigns, and determining if there are notable differences between them. We will look separately at users supporting different parties, with a special attention towards Podemos and IU, the parties that lost more votes.

\section{Dataset}

This study relies on two different datasets collected from Twitter in relation to the electoral campaigns of the 2015 and 2016 Spanish national elections (December 4-20 2015 and June 10-26 2016). Tweets were collected if they either (a) 
Table 1: Number of nodes $\left(N_{2015}\right.$ and $\left.N_{2016}\right)$ and edges $\left(E_{2015}\right.$ and $\left.E_{2016}\right)$ for the intra-network of each cluster in the retweet networks of 2015 and 2016.

\begin{tabular}{l|rr|rr} 
Cluster & $N_{2015}$ & $E_{2015}$ & $N_{2016}$ & $E_{2016}$ \\
\hline Podemos & 16114 & 33488 & 9771 & 12818 \\
IU & 10439 & 22422 & 10314 & 12304 \\
PP & 8345 & 28677 & 5614 & 11682 \\
PSOE & 7538 & 25119 & 5541 & 10174 \\
CS & 7200 & 24110 & 5458 & 9501 \\
ECP & 1412 & 2925 & 1791 & 2868
\end{tabular}

were created by, (b) retweeted or (c) mentioned one of the official party accounts or party candidate accounts (listed in Table B.1).

To detect the Twitter organization of political parties, we build directed weighted graphs of users (nodes) and retweets (edges). Each weighted edge indicates the number of times the source user retweeted a message posted by the target user. We filter edges with weights lower than 3 to exclude anecdotal interactions as in 2]. The resulting network characteristics for 2015 and 2016 are presented in Table B.2.

\section{Network Analysis}

We start showing some general results about our community discovery analysis for both election campaigns. We then analyze how the found clusters change between the two elections and conclude this section with a quantification of the change in political engagement.

\subsection{Community detection}

Table 1 shows the clustering results obtained using the N-Louvain method 9 in both networks, showing only the largest clusters. For the four parties that formed the coalition -Podemos, En Comú Podem (ECP), En Marea and Compromís-, only two clusters are identified, corresponding to Podemos and ECP. The others, En Marea and Compromís, are effectively integrated in Podemos, while ECP forms a separate cluster. This separate cluster might be explained by the use of different languages (Catalan instead of Spanish). We also observe that the IU party maintains its cluster despite merging with Podemos in the second election. The IU cluster is slightly bigger than the one of Podemos in the second election. This is noteworthy, since Podemos had by far a larger amount of votes in the first election, and one might expect the opposite effect in the network. In general, we conclude that the formation of the coalition for the 2016 elections is not captured by the observed communities, since Podemos, IU and ECP are associated to different clusters. Moreover, there is no obvious relation between the size of the identified communities and the electoral results, in terms of votes.

\footnotetext{
${ }^{9}$ See Appendix A.1 for a description of the N-Louvain method.
} 

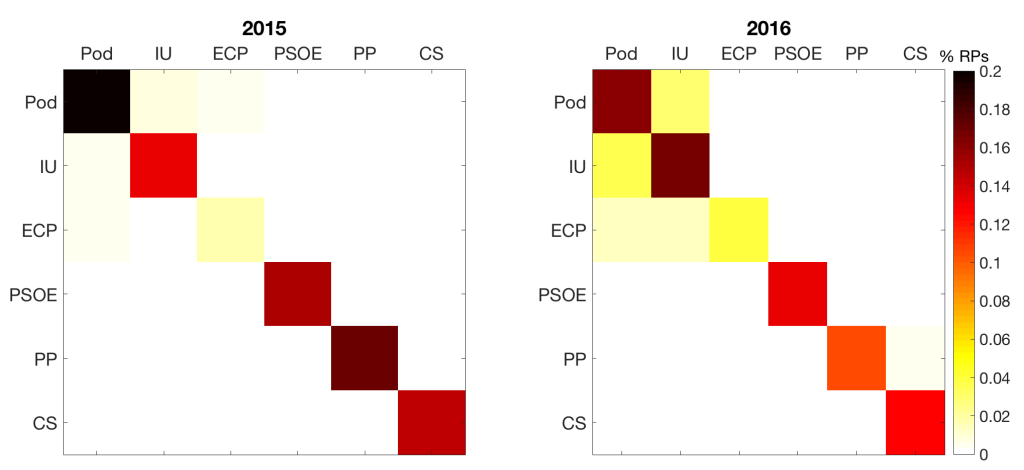

Fig. 1: Normalized weighted adjacency matrices of the 2015 (left) and 2016 (right) retweet networks aggregating nodes by party clusters.

The analysis of the inter-cluster and intra-cluster density of edges provides a measure of how strongly are the interactions within and between the different parties in the different campaigns. Table 1 (second and fourth columns) shows that the amount of intra-cluster edges is smaller in 2016 than in 2015, with a decrease by almost $50 \%$, indicating weaker connections in the second election.

What about the inter-cluster edges? We would expect some of these interactions to increase in the second election, as a consequence of the electoral coalition and of the synergies between the parties. To examine all the interactions between the parties, we consider the interaction matrix $A$, where $A_{i j}$ is the normalized sum of all retweets that users from cluster $i$ made for the tweets from users of cluster $j$ displayed in Figure 1 for both elections. Both matrices are diagonally dominant, since the vast majority of retweets were made between users from the same cluster in both elections, being this behavior more pronounced in 2015 than in 2016. Comparing the parties involved in the coalition, we clearly observe that their interactions increased in 2016, as the (yellow) off-diagonal elements indicate. Interestingly, the interaction between ECP and the other members of the coalition is not symmetric. This fact may be explained again by linguistic reasons, since ECP users retweet both messages in Spanish and Catalan, but most users in Podemos and IU clusters only speak Spanish and therefore do not retweet ECP messages in Catalan. We conclude that, despite the coalition is not captured at the clustering level (parties within the coalition do not merge into a single cluster), it is captured at the level of the interactions between clusters, which increased remarkably in 2016.

\subsection{Cluster dynamics between the two elections (RQ1)}

We now analyze how the clusters change between campaigns. Table B.4 shows some general indicators. We observe that all but a single cluster (ECP) shrink in the second campaign (negative balance), indicating a significant decrease in 


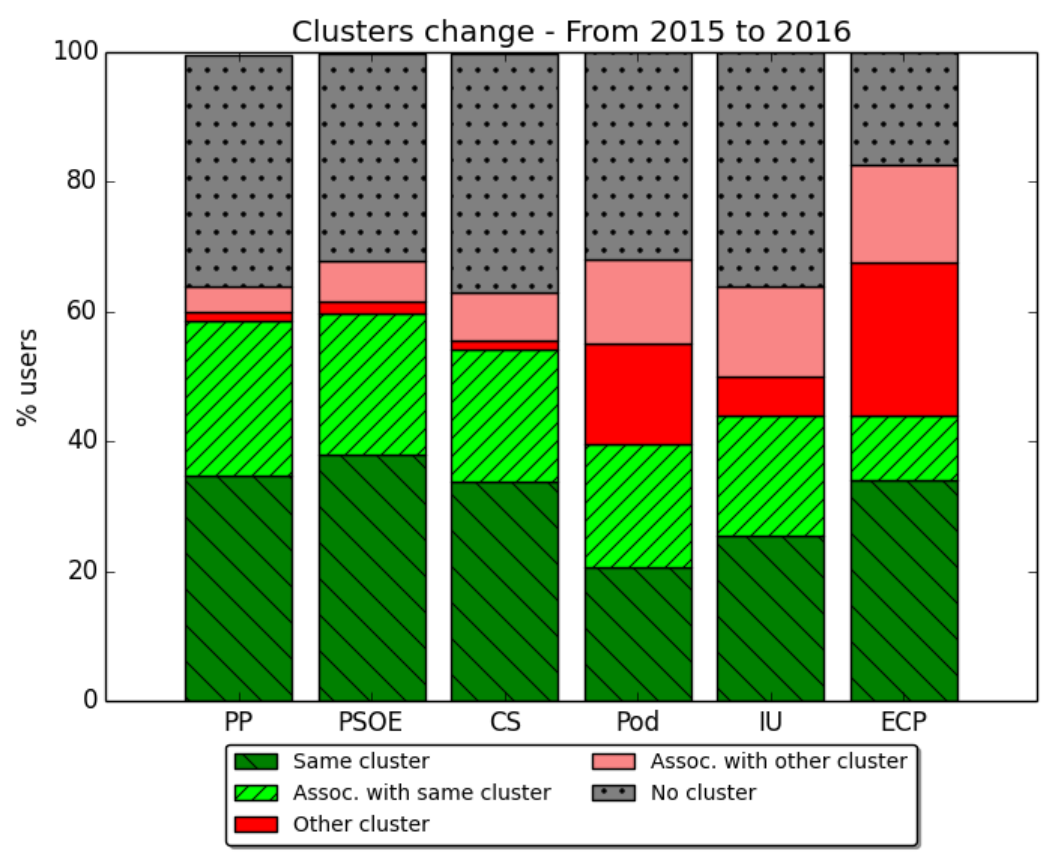

Fig. 2: Proportion of clustered users in 2015 who in 2016: remain in the same cluster; retweet mostly users from the same cluster (associated with same cluster); lie in another cluster; retweet mostly users from another cluster (associated with other cluster); are not associated to any cluster (no cluster).

activity and suggesting an overall decrease in motivation. Another important observation is that all clusters lose more than half of the users they had in 2015. The cluster that loses less users is PSOE (62\%) while the one that loose more is Podemos (nearly 80\%). This illustrates the high variability between the users assigned to the clusters in both campaigns. Podemos has the highest negative balance among all clusters, losing 6324 users. Notice that IU, although being apparently the most stable cluster, is actually the second one that lost more members. The balance is explained because it also gained many new users from other clusters. Looking at the joint clusters of Unidos Podemos (UP), we see that it suffers a higher loss than the parties not in the coalition (69.6\% of UP vs $62 \%-66 \%$ ), indicating that not all the users migrate within the parties of the coalition.

To understand how users migrate between clusters, we apply the methodology described in A.2. Figure 2 shows the distribution of the different categories of users for each party cluster in 2015, providing a more detailed view of the (lost) users of Table B.4 who correspond to the non dark-green regions in the figure. We analyze first the distributions of PP, PSOE and CS. They follow a similar 
pattern with around $35 \%$ of users remaining in the same cluster and around $25 \%$ of users associated with the same cluster. Despite losing the majority of users according to our clustering criteria, approximately $60 \%$ of their users do not change their support in 2016 . The remaining $\approx 40 \%$ either do not have a cluster assigned in 2016 or by a small percentage migrated to other parties.

Regarding the UP clusters, Podemos has the smallest number of stable supporters (dark/light green) and ECP is the one with smallest proportion of users who do not fall in any cluster in 2016 (gray), which indicates that ECP users keep a high activity in the 2016 campaign. When viewed independently, Podemos, IU and ECP have a smaller proportion of users that stay in the same cluster or are associated to same cluster (dark/light green) than the other parties. However, when considered together in UP, this proportion increases and becomes comparable to the other parties. This suggests that migrations occur mostly within the clusters of the UP coalition. This is confirmed in Figure B.1, which shows the flow of clustered users between campaigns for the users clustered in both elections (either in the same cluster or in another cluster). It is noticeable that most of these users fall in the same party in both elections, indicating a strong political association. Clearly, Podemos is the cluster that suffers more changes, with a considerable amount of users that mostly migrate to IU and, to a lesser extent, to ECP. We do not see the same behavior in IU.

The following conclusions are extracted from the entire analysis on cluster changes: although the large variability observed initially in the compositions of the clusters, when the associated users are considered as well, the migration of parties is reduced, with exceptions within Unidos Podemos. It seems that users who actively participate in Twitter are usually very positioned towards one party and only retweet other parties very sporadically. In general, users that retweet the messages of a party tend to either keep supporting the same party, or stop participating actively in the campaign.

Unidos Podemos is the entity that loses more support from the first to the second election, as Table B.4 and Figures 2 and B.1 show. The total balance between the two elections is negative and stronger than for the rest of the parties. However, when analyzing the nature of the cluster in 2016 and its changes in relation to 2015, this negative balance is not as high as expected from the electoral results (in relation to the other parties) and it does not seem to reflect the general demotivation which was interpreted from the electoral results. In Unidos Podemos, we have seen a strong migration of supporters from Podemos to IU, which did not happen in the opposite direction. The Spanish electoral law that favors bigger parties may have had an influence, pushing citizens closer to IU to vote and campaign for the bigger party Podemos in 2015.

\subsection{Political engagement (RQ2)}

Twitter activity can be an indicator of political engagement. To characterize the activity of users in each cluster and in each election, we calculate the cumulative distribution function, or probability $P(X \leq x)$ that a user $X$ retweets less than or $x$ times, for those users that where present in both campaigns. Results are 


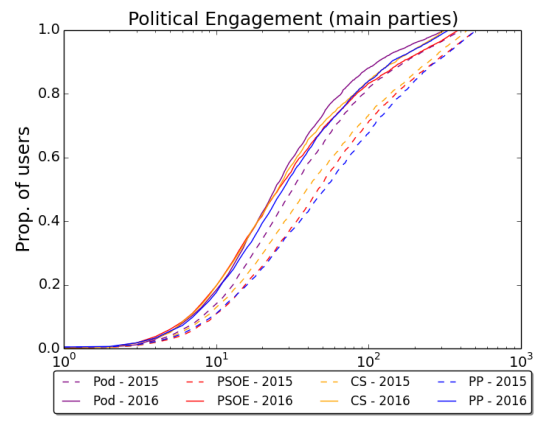

(a) Podemos, PP, PSOE and CS.

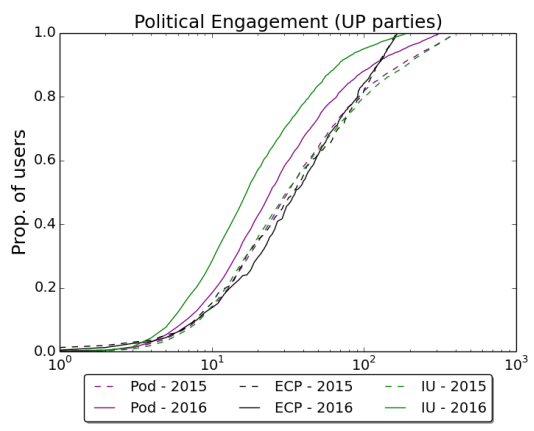

(b) UP clusters: Podemos, IU and ECP

Fig. 3: Cumulative distribution of the number of tweets per users who fall in the same party cluster in 2015 (dashed) and 2016 (continuous).

displayed for PP, PSOE, CS and Podemos in Figure 3a. The solid curves (activity in 2016) lie above the dashed ones (activity in 2015), indicating a decrease of activity for all parties. These results confirm that political engagement decreased, perhaps due to the user fatigue after a long period of political activity.

To analyze the engagement within the different parties that form the UP coalition, we break down the coalition and show in Figure $3 \mathrm{~b}$ the cumulative distribution functions for each UP cluster individually. First, we observe that all curves show a similar profile in 2015. However, in 2016 the picture changes. We observe that IU has much less activity than Podemos. Since our analysis includes the strongest supporters of the party only, a decrease in their activity suggests that those users might have been unhappy with the coalition and were demotivated during the second election. This result is in agreement with previous literature [20] and with the post-electoral study from Metroscopia [10], which reported that the UP coalition retained only three out of four Podemos voters $(74 \%)$ and only six out of ten IU voters (60\%). Moreover, the Catalan ECP shows the opposite effect than the rest of parties (it actually increases its activity in the second election), also in agreement with the electoral results in Catalonia, where Unidos Podemos lost fewer voters.

We can conclude that our proposed methodology that analyzes the activity distribution within the obtained clusters satisfactorily captures the observed behaviour with respect to engagement observed in the election. 


\section{Discussion}

We have presented a methodology to analyze online Twitter campaigns based on several steps. First, we have used a robust community discovery method and matched automatically the user clusters across multiple executions of the Louvain method using the Jaccard coefficient. Second, we have proposed a characterization of the cluster composition dynamics in consecutive elections to reflect changes in party inclinations. Finally, we have analyzed political engagement by means of the Twitter activity distributions in the different clusters. Our proposed methodology can be seen as a refinement of similar approaches proposed recently for the analysis of online Twitter campaigns [2].

The analysis of user migration between party clusters (RQ1) reveals that several users have transitioned within the coalition. The results expose an important transfer of users associated to Podemos in the 2015 election to the cluster of Izquierda Unida in 2016. Those users might have been supporting the bigger party in the first election as a matter of utility when it came to getting representatives while actually feeling closer to the smaller party. The results also show a smaller proportion of users who remain in the UP clusters in 2016 compared to other parties, which may reflect the demotivation of its electorate, although this signal is weak compared to the large decrease in votes for UP.

Previous research has indicated how Twitter activity may be thought as an indicator of political engagement of the users [3] $9[12$. Our study has also analyzed whether there is a relation between the motivation of the electorate and activity on Twitter (RQ2). Despite our analysis shows a lower activity in 2016 than in 2015 for all mayor parties, in line with the participation decrease, the results follow a very similar pattern for all parties although the electoral results were different for them. Moreover, the decrease in activity is not significantly higher for the users in the UP clusters, which lost the highest amount of votes. However our analysis reveled differences within the UP clusters, showing a much larger decay in activity for IU supporters. This may indicate that users strongly associated to IU were less appealed by the coalition, in agreement with existing studies [20]10].

\section{Acknowledgments}

This work is supported by the Spanish Ministry of Economy and Competitiveness under the María de Maeztu Units of Excellence Programme (MDM-2015-0502).

\section{Appendix}

\section{A Methods}

\section{A.1 N-Louvain method}

The Louvian method [4] is widely used as a community detection algorithm because it is efficient and finds the correct clustering in certain types of networks. 
However, some care needs to be taken when applying this algorithm in our context. In particular, since the algorithm has a random component, different executions may typically produce different partitions for the same network. To obtain robust results and find a reliable cluster assignment, we follow the method introduced in 2, which performs multiple executions of the Louvain algorithm and only considers nodes that fall almost all the times into the same cluster.

To identify each cluster across executions, we improve the previous method by applying the Jaccard index [13] to every pair of clusters $c_{i}$ and $c_{j}$ across different executions:

$$
J\left(c_{i}, c_{j}\right)=\frac{\left|c_{i} \cap c_{j}\right|}{\left|c_{i} \cup c_{j}\right|} .
$$

Thus, clusters across executions are matched if they are the most similar ones. This allows us to assess the proportion of times a node falls within the same cluster. Finally, the method assigns to each cluster all the nodes that appear in that cluster in at least a fraction $(1-\varepsilon)$ of the partitions created, that is to say, $\varepsilon$ represents the sensibility level of the algorithm $(\varepsilon=0.05$ in this study). This procedure allows to validate the results of the community detection algorithm and to guarantee that all the nodes that are assigned to a cluster do actually belong to it with a given confidence. The remaining nodes, that cannot be assigned in a stable way to any of the main clusters, are left out from all the clusters.

\section{A.2 Cluster changes between networks}

To characterize how users change between two consecutive networks, $G_{1}$ and $G_{2}$, we consider five possible categories, depending on how a user $i$ that belongs to a cluster in $G_{1}$ is related to the clustering in $G_{2}$. Let $c_{1}(i)$ and $c_{2}(i)$ denote the cluster to which $i$ belongs in $G_{1}$ and $G_{2}$, respectively. There are three main possible scenarios, either the user belongs to the same cluster in both networks,

1. $c_{1}(i)=c_{2}(i)$ (Same cluster),

2. it belongs to different clusters, $c_{1}(i) \neq c_{2}(i)$ (Other cluster),

3 . or $i$ does not fall robustly in any cluster of $G_{2}$. In this case, we can still assign a cluster to $i$ depending on whether:

(a) $i$ retweeted users belonging to the same cluster $c_{1}(i)$ (we call this category Associated with same cluster), or

(b) $i$ retweeted users belonging to another cluster (Associated with other cluster).

(c) Finally, if the level of activity of $i$ does not reach the threshold to be included in $G_{2}$ (we only include interactions that occur at least three times), we assign $i$ to the category None. 


\section{B Additional Tables and Figures}

Table B.1: Twitter accounts of the selected political parties and candidates which were used to retrieve the datasets.

\begin{tabular}{|c|c|c|}
\hline Party & Party account & Candidate account \\
\hline $\mathrm{PP}$ & @PPopular & @marianorajoy \\
\hline PSOE & $@$ @SOE & @sanchezcastejon \\
\hline Podemos & @ahorapodemos & @Pablo_Iglesias_ \\
\hline IU & @iunida & @agarzon \\
\hline C's & @CiudadanosCs & @Albert_Rivera \\
\hline En Comú Podem & @EnComu_Podem & @XavierDomenechs \\
\hline Compromís & @compromis & @joanbaldovi \\
\hline Equo & $@$ Equo & $@$ juralde \\
\hline Marea-Anova-EU & @En_Marea & @tone_corunha \\
\hline ERC-CATSÍ & @Esquerra_ERC & @gabrielrufian \\
\hline DL & $@$ ConvergenciaCAT & @franceschoms \\
\hline EAJ-PNV & @eajpnv & @MikelLegarda \\
\hline Bildu & @ehbildu & @ikerurbina1 \\
\hline CCa-PNC & @gnacionalista & @PabloRodriguezV \\
\hline
\end{tabular}

Table B.2: Retweet network indicators for 2015 and 2016: number of retweets for the whole election (\# tweets), number of nodes (N) and edges (E) in the network, clustering coefficient $(\mathrm{cl})$ and average path length $(\ell)$.

\begin{tabular}{l|ccccl} 
Elections of & \# tweets & N & E & cl & \multicolumn{1}{c}{$\ell$} \\
\hline 2015 & 3196677 & 57575 & 164411 & 0.004 & 7.18 \\
2016 & 1602528 & 72269 & 168135 & 0.0015 & 6.215
\end{tabular}


Table B.3: Participation, percentage of obtained votes and parliament seats per party for the 2015 and 2016 elections. Pod+ stands for the sum of Podemos, En Comú Podem, En Marea, and Compromis. In 2016 IU is added to this sum as well.

\begin{tabular}{c|c|cccccc} 
Election & Participation & PP & PSOE & Pod+ & IU & CS & Other \\
\hline 2015 & $69.67 \%$ & $28.71 \%$ & $22.01 \%$ & $20.4 \%$ & $3.68 \%$ & $13.94 \%$ & $11.26 \%$ \\
& & 123 & 90 & 69 & 2 & 40 & 26 \\
\hline 2016 & $66.48 \%$ & $33.01 \%$ & $22.63 \%$ & $20.79 \%$ & $13.05 \%$ & $10.52 \%$ \\
& & 137 & 85 & & 71 & 32 & 25
\end{tabular}

Table B.4: Main clusters per party. In columns: cluster sizes in 2015 and 2016, \# of users present in the cluster in 2015 but not in 2016 (lost) and the corresponding percentage, \# of users present in the cluster in 2016 but not in 2015 (new), difference (balance) between new and lost users. Last line (UP) is the sum of ECP, Podemos and IU

\begin{tabular}{l|r|r|rl|r|r} 
Cluster & size 2015 & size 2016 & lost & new & balance \\
\hline CS & 7200 & 5458 & 4771 & $(66.3 \%)$ & 3029 & -1742 \\
PP & 8345 & 5613 & 5446 & $(65.3 \%)$ & 2714 & -2732 \\
PSOE & 7538 & 5541 & 4674 & $(62.0 \%)$ & 2677 & -1997 \\
\hline ECP & 1412 & 1791 & 930 & $(65.9 \%)$ & 1309 & 379 \\
Podemos & 16113 & 9771 & 12806 & $(79.5 \%)$ & 6464 & -6342 \\
IU & 10439 & 10313 & 7792 & $(74.6 \%)$ & 7666 & -126 \\
\hline UP & 27964 & 21875 & 19448 & $(69.6 \%)$ & 13359 & $\mathbf{- 6 ~ 0 8 9}$
\end{tabular}

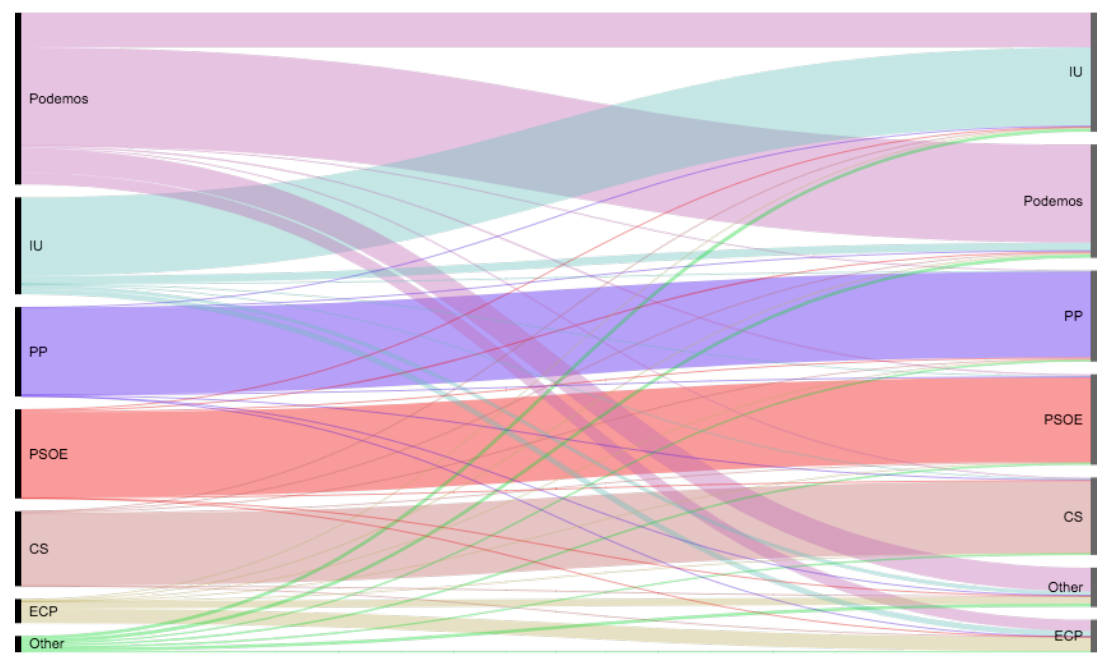

Fig. B.1: Redistribution of cluster users: amount of users from a 2015 cluster (left) in the 2016 clusters (right). 


\section{References}

1. Aragón, P., Kappler, K.E., Kaltenbrunner, A., Laniado, D., Volkovich, Y.: Communication dynamics in Twitter during political campaigns: The case of the 2011 spanish national election. Policy \& Internet 5(2), 183-206 (2013)

2. Aragón, P., Volkovich, Y., Laniado, D., Kaltenbrunner, A.: When a Movement Becomes a Party: Computational Assessment of New Forms of Political Organization in Social Media. In: ICWSM-16 - 10th International AAAI Conference on Web and Social Media. The AAAI Press (2016)

3. Bakker, T.P., De Vreese, C.H.: Good news for the future? young people, Internet use, and political participation. Communication research 38(4), 451-470 (2011)

4. Blondel, V.D., Guillaume, J.L., Lambiotte, R., Lefebvre, E.: Fast unfolding of communities in large networks. Journal of Statistical Mechanics: Theory and Experiment 2008(10), P10008 (2008)

5. Caldarelli, G., Chessa, A., Pammolli, F., Pompa, G., Puliga, M., Riccaboni, M., Riotta, G.: A multi-level geographical study of Italian political elections from Twitter data. PloS one 9(5), e95809 (2014)

6. Castells, M.: The new public sphere: Global civil society, communication networks, and global governance. The aNNalS of the american academy of Political and Social Science 616(1), 78-93 (2008)

7. Chung, J.E., Mustafaraj, E.: Can collective sentiment expressed on Twitter predict political elections? In: AAAI. vol. 11, pp. 1770-1771 (2011)

8. Conover, M., Ratkiewicz, J., Francisco, M., Gonçalves, B., Menczer, F., Flammini, A.: Political polarization on Twitter. In: ICWSM (2011)

9. Dimitrova, D.V., Shehata, A., Strömbäck, J., Nord, L.W.: The effects of digital media on political knowledge and participation in election campaigns: Evidence from panel data. Communication Research 41(1), 95-118 (2014)

10. Ferrándiz, J.P.: Fidelidades y fugas para explicar los resultados del 26j (2016), http://metroscopia.org/ fidelidades-y-fugas-para-explicar-los-resultados-del-26j/] [Online; accessed 10-June-2017]

11. Gayo-Avello, D.: No, you cannot predict elections with Twitter. IEEE Internet Computing 16(6), 91-94 (2012)

12. Holt, K., Shehata, A., Strömbäck, J., Ljungberg, E.: Age and the effects of news media attention and social media use on political interest and participation: Do social media function as leveller? European Journal of Communication 28(1), 19-34 (2013)

13. Jaccard, P.: Etude comparative de la distribution florale dans une portion des Alpes et du Jura. Impr. Corbaz (1901)

14. Jungherr, A.: Twitter use in election campaigns: A systematic literature review. Journal of information technology \& politics 13(1), 72-91 (2016)

15. Jungherr, A., Jürgens, P., Schoen, H.: Why the pirate party won the german election of 2009 or the trouble with predictions: A response to tumasjan, a., sprenger, to, sander, pg, \& welpe, im "predicting elections with Twitter: What 140 characters reveal about political sentiment". Social science computer review 30(2), 229-234 (2012)

16. Llaneras, K.: Qué votantes cambiaron su voto el 26-j (2016), http://politica. elpais.com/politica/2016/07/22/ratio/1469195845_977293.html, [Online; accessed 10-June-2017 ] 
17. Metaxas, P.T., Mustafaraj, E.: Social media and the elections. Science 338(6106), 472-473 (2012)

18. Orriols, L., Cordero, G.: The breakdown of the Spanish two-party system: the upsurge of Podemos and Ciudadanos in the 2015 general election. South European Society and Politics pp. 1-24 (2016)

19. Peña-López, I., Congosto, M., Aragón, P.: Spanish Indignados and the evolution of the $15 \mathrm{M}$ movement on Twitter: towards networked para-institutions. Journal of Spanish Cultural Studies 15(1-2), 189-216 (2014), http://dx.doi.org/10.1080/ 14636204.2014 .931678

20. Simón, P.: The challenges of the new Spanish multipartism: government formation failure and the 2016 general election. South European Society and Politics 21(4), 493-517 (2016)

21. Tumasjan, A., Sprenger, T.O., Sandner, P.G., Welpe, I.M.: Predicting elections with Twitter: What 140 characters reveal about political sentiment. ICWSM 10(1), 178-185 (2010)

22. Vergeer, M., Hermans, L.: Campaigning on Twitter: Microblogging and online social networking as campaign tools in the 2010 general elections in the netherlands. Journal of Computer-Mediated Communication 18(4), 399-419 (2013) 